\title{
A first look at colloid-associated trace metals of AMD-influenced waters in a short-rotation forestry field experiment
}

SARAH NETTEMANN, DANIEL MIRGORODSKY, KEVIN

LENK, ERIKA KOTHE AND THORSTEN SCHÄFER

Friedrich Schiller University Jena

Presenting Author: sarah.nettemann@uni-jena.de

Heavy metal (HM) and radionuclide ( $\mathrm{RN})$ transport in soils and groundwater systems is facilitated by association with mobile inorganic or organic natural nanoparticles (NPs) [1]. The BMBF-funded (Federal Ministry of Education and Research) project USER-II aims to optimize bioremediation strategies combined with bioenergy production on areas moderately contaminated with $\mathrm{HM}$ and $\mathrm{RN}$. For this, the test field "Gessenwiese" was established at a former Uranium mining area near Ronneburg, Thuringia, Germany [2]. In 2016, birch, alder, and willow trees were planted within short-rotation forestry. Soil amendments such as inoculation with mycorrhiza and streptomyces and the addition of calciferous soil (rendzina) are tested and compared to control plots. The remediation efforts have led to an increase in soil $\mathrm{pH}$ and to a decrease in the fraction of mobile $\mathrm{HM} / \mathrm{RN}$ in the soil material. In previous years, pore- and groundwater samples were analyzed regarding their contents of "dissolved" HM and RN, operationally defined as the $<0.45 \mu \mathrm{m}$ fraction.

This work aims to elucidate the mobility of colloid-associated trace metals in the $<0.45 \mu \mathrm{m}$ fraction of pore- and groundwater samples. Additionally, a special emphasis is placed on the characterization and quantification of organic compounds and nitrogen species using a Liquid Chromatography - Organic Carbon Detection - (Organic) Nitrogen Detection system (LCOCD-OND).

First analyses show high variations in the concentration of organic carbon and nitrogen compounds, nitrate, and ammonium in the hydrogeological year. Nanoparticle Tracking Analysis (NTA) results of water sampled during 2020 revealed NP concentrations between $3.2 \mathrm{E}+06 \pm 8.1 \mathrm{E}+05$ particles $\mathrm{ml}^{-1}$ and $8.3 \mathrm{E}+09 \pm 3.5 \mathrm{E}+08$ particles $\mathrm{ml}^{-1}$ and a mean hydrodynamic diameter of $162 \pm 36 \mathrm{~nm}(\mathrm{n}=61)$. These findings highlight the importance to further investigate the colloid-associated transport of $\mathrm{HM}$ and $\mathrm{RN}$ in this area. Furthermore, operational fractions derived from LC-OCD-OND will be analyzed with ICP-MS to elucidate specific HM or RN associations. First results on the role of the inoculation and root exudates on the HM/RN speciation will be presented.

[1] Kretzschmar \& Schäfer (2005), Elements 1, 205-210.

[2] Grawunder et al. (2009), Chemie Der Erde - Geochemistry 69, 5-19. 